\title{
Wind buffeting of large telescopes
}

\author{
Douglas G. MacMynowski ${ }^{1, *}$ and Torben Andersen ${ }^{2}$ \\ ${ }^{1}$ Control and Dynamical Systems, California Institute of Technology, 1200 East California Boulevard, \\ Pasadena, California 91125, USA \\ ${ }^{2}$ Lund Observatory, Lund University, Box 43, SE-221 00 Lund, Sweden \\ ${ }^{*}$ Corresponding author: macmardg@cds.caltech.edu
}

Received 4 November 2009; accepted 3 December 2009;

posted 22 December 2009 (Doc. ID 119505); published 26 January 2010

\begin{abstract}
Unsteady wind loads due to turbulence within the telescope enclosure are one of the largest dynamic disturbances for ground-based optical telescopes. The desire to minimize the response to the wind influences the design of the telescope enclosure, structure, and control systems. There is now significant experience in detailed integrated modeling to predict image jitter due to wind. Based on this experience, a relatively simple model is proposed that is verified (from a more detailed model) to capture the relevant physics. In addition to illustrating the important elements of the telescope design that influence wind response, this model is used to understand the sensitivity of telescope image jitter to a wide range of design parameters. (C) 2010 Optical Society of America
\end{abstract}

OCIS codes: $110.6770,120.7280$.

\section{Introduction}

Understanding the effect of unsteady wind forces on telescope structures during the design phase has long been the subject of research [1,2]. This is particularly relevant as telescopes get larger, while the desired performance gets more stringent. The current design efforts for extremely large telescopes have resulted in substantial progress in understanding the wind environment within the telescope enclosure [3-7] and in detailed integrated modeling to understand the effects of wind on performance [8-10].

These integrated models include (i) a structural model obtained from finite element analysis that includes many degrees of freedom, (ii) an optical model, either directly employing ray tracing or a linear model obtained from prior ray tracing, (iii) a description of the wind forces, e.g., from wind-tunnel data or computational fluid dynamics (CFD) or a parameterized model informed by CFD, and (iv) a control model for the relevant degrees of freedom. These models are essential for understanding which effects are important, and to what extent. However, based on the ex-

0003-6935/10/040625-12 $\$ 15.00 / 0$

(C) 2010 Optical Society of America perience that has been gained from these efforts, and from the Thirty Meter Telescope (TMT) integrated modeling in particular, a much simpler model is given here that captures the relevant physical processes involved in determining the image jitter response for a large optical telescope. The wind also results in image degradation due to optics misalignment (typically small) and from the motion of the primary mirror (M1) segments caused by forces on M1. While this latter response may be significant [10], we focus on image jitter.

In addition to documenting the achieved understanding about how to predict the wind response of large ground-based telescopes, the simpler description can be used to understand the parametric sensitivity of the response to choices in wind-loading parameters (external wind speed, dome reduction factor, outer scale of turbulence, and spatial correlation length), structural design parameters (crosssectional areas, moment arm about the elevation axis, inertia, and quasi-static structural flexibility), and key control parameters (mount control and optical guide loop bandwidths).

The approach taken to model wind response of the Very Large Telescope [2] included a von Karman wind model and an aerodynamic attenuation factor, 
and the response due to the finite mount control bandwidth was computed. Padin and Davison [11] added both structural dynamic amplification and the response of M1 due to loads on the top end structure. Neither of these latter effects should be significant for a well-designed structure, although their analysis was essential to ensuring that this is true for future telescopes.

While the broad approach here is similar in some ways to these earlier parametric models, the understanding of which physical processes matter, and which can be neglected, can now be made on the basis of the full integrated models, and the description herein thus differs in several ways. The description of wind forces on the telescope is now informed by substantial wind-tunnel and CFD analyses (see [4] in particular). The wind description also includes a more thorough analysis of the spatial coherence of the pressure than has been used in previous studies (see Subsection 2.A). This allows an accurate treatment of the forces on long slender structural members supporting the top end of the telescope (M2 and any additional components); the aerodynamic attenuation factor typically applied, e.g., in [2] is only appropriate for low-aspect-ratio structures. The full integrated model demonstrates that the dominant contribution to image jitter is due to residuals from the mount control system (MCS), and typically the elevation axis in particular. The quasi-static structural flexibility contributes slightly, but dynamic amplification from structural resonances is negligible. A low-bandwidth guide loop is sufficient to make the structural contribution small compared to the mount control contribution to image jitter. The response can therefore be estimated by (i) estimating the spectrum of torque disturbances about the elevation axis, (ii) computing the torque response for the elevation-axis MCS, and (iii) multiplying the spectrum and response and integrating.

The emphasis here is on understanding the relevant processes and parameters to predict the response, given a set of input parameters, and not on the specific values of the parameters. These will clearly vary as a function of the design. TMT parameters are used herein only as a design point, at which a more detailed model exists that is used to validate the simple approach.

The next section describes the estimation of wind forces and the telescope response. This model is justified in Section 3 by comparing the response to the predictions made by a full integrated model. Section 4 then uses the model to explore how the response varies for a wide range of parameters.

\section{Modeling}

\section{A. Wind Force from Velocity}

Before discussing wind-loading assumptions specific to telescopes, it is useful to first clarify the relationship between wind speed and force, and in particular the resulting spectrum, including spatial decorrelation effects.

The instantaneous velocity $u$ can be decomposed into its mean $\bar{u}$ and unsteady component $u^{\prime}$, with $u_{\text {rms }}$ as the root mean square (rms) of the unsteady velocity, and turbulence intensity $\mathcal{I}=u_{\mathrm{rms}} / \bar{u}$. The dynamic pressure is proportional to $u^{2}=\left(\bar{u}+u^{\prime}\right)^{2}$, and by subtracting off the mean, the unsteady component of pressure is proportional to

$$
u^{2}-\overline{u^{2}}=2 \bar{u} u^{\prime}+\left(u^{\prime 2}-\overline{u^{\prime 2}}\right) .
$$

If $u^{\prime}$ is normally distributed, then the rms of $u^{2}-\overline{u^{2}}$ can be expressed as the square of an "effective" velocity [4]]:

$$
u_{\mathrm{eff}}^{2}=\left[\left(2 \bar{u} u_{\mathrm{rms}}\right)^{2}+2 u_{\mathrm{rms}}^{4}\right]^{1 / 2}=2 \mathcal{I} \bar{u}^{2}\left(1+\mathcal{I}^{2} / 2\right)^{1 / 2},
$$

and the rms unsteady pressure is then $p_{\text {rms }}=\frac{1}{2} \rho u_{\text {eff }}^{2}$, where $\rho$ is the air density. The second term in Eqs. (1) and (2) is often ignored, which is a reasonable approximation even for a turbulence intensity of order 0.5.

Typically, von Karman spectra are assumed; this is well validated for turbulence inside the telescope enclosure [4]. The von Karman spectrum for velocity [12] as a function of temporal frequency $f$ can be written as

$$
\begin{aligned}
\Phi_{u}(f) & =u_{\mathrm{rms}}^{2}\left(\frac{\sqrt{3} \Gamma(2 / 3) \Gamma(5 / 6)}{\pi^{3 / 2}}\right) \frac{L_{0} / \bar{u}}{\left[1+\left(L_{0} f / \bar{u}\right)^{2}\right]^{5 / 6}} \\
& =u_{\mathrm{rms}}^{2} \frac{4 L_{u} / \bar{u}}{\left[1+70.74\left(L_{u} f / \bar{u}\right)^{2}\right]^{5 / 6}}
\end{aligned}
$$

where normalizing the spectrum leads to either the expression involving Gamma functions or the factor of 70.74 (see, e.g., [13]). Both $L_{0}$ in ()ㅡㄹ and $L_{u}$ in () have been referred to as the "outer scale of turbulence." The former is consistent with general usage in turbulence modeling, and that is the definition we follow herein. ( $L_{u}$ is an average correlation length, not the largest scale.)

The Kolmogorov scaling arguments that lead to the $-5 / 3$ exponent in the frequency dependence of the velocity spectrum give a $-7 / 3$ exponent for pressure [14]. Introducing the outer scale in the same manner as for the von Karman velocity spectrum yields the (normalized) pressure spectrum:

$$
\Phi_{p}(f)=p_{\mathrm{rms}}^{2}\left(\frac{2 \sqrt{\pi}}{3 \Gamma(2 / 3) \Gamma(5 / 6)}\right) \frac{L_{0} / \bar{u}}{\left[1+\left(L_{0} f / \bar{u}\right)^{2}\right]^{7 / 6}} .
$$

It is common to assume that the pressure spectrum has the same $-5 / 3$ exponent as the velocity spectrum; this could be inferred by neglecting the final 
term in (1). However, this incorrect assumption shifts a greater fraction of the energy to higher frequencies. Note that Eq. (5) is experimentally verified, e.g., in measurements on the Gemini primary mirror [4].

The force is obtained by integrating pressure over a surface of area $A$. At low frequencies, the unsteady pressure is spatially correlated over long distances, while at higher frequencies, the spatial correlation length decreases. It is essential to include this effect in modeling the wind force. A final slope of $-13 / 3$ for the force spectrum has been observed in windtunnel force measurements on low-aspect-ratio bluff bodies [13].

The force spectrum is given by the product of the pressure spectrum in Eq. (5) and a frequencydependent spatial decorrelation factor $H(f)$ (see Eqs. (8) and (9), for example). This factor can be derived by integrating the pressure over the area to obtain force (see Appendix A):

$$
H(f)=\left[\frac{1}{1+\left(f / f_{x}\right)^{2}} \times \frac{1}{1+\left(f / f_{y}\right)^{2}}\right]^{1 / 2} \text {. }
$$

This is equivalent to adding $n$ independent forces in quadrature, where $n$ is the ratio of the length of the structure to the frequency-dependent correlation length. The characteristic frequencies $f_{x}$ and $f_{y}$ depend on the local mean wind speed and the projected length and width of the structural member $\ell_{x}$ and $\ell_{y}$ as $f_{x}=\bar{u} /\left(\gamma \ell_{x}\right), f_{y}=\bar{u} /\left(\gamma \ell_{y}\right)$. The value $\gamma=2$, derived in AppendixA A for a 1-D structure, corresponds to a correlation length of half the wavelength.

A common (but not technically correct) approach is to describe the pressure spectrum using the $-5 / 3$ Kolmogorov exponent for the velocity spectrum as noted above, and then to still obtain the correct $-13 / 3$ final slope for the force spectrum, this is multiplied by the empirically derived aerodynamic attenuation factor $[\underline{2}, \underline{13}, \underline{15}]$ :

$$
\chi^{2}(f)=\left[\frac{1}{1+(2 f \sqrt{A} / \bar{u})^{4 / 3}}\right]^{2} .
$$

For low-aspect-ratio bluff bodies for which $\ell_{x} \simeq$ $\ell_{y} \simeq \sqrt{A}$, then the product of the von Karman pressure spectrum and decorrelation $H(f)$ is similar to the product obtained using the spectrum with the Kolmogorov exponent for velocity and the aerodynamic attenuation factor in Eq. (7). However, for high-aspect-ratio structural members relevant in telescope wind analysis, the different decorrelation in different directions matters; this will be shown in the example in Subsection 3.D.

Note that both the original measurements and the theory underlying Eq. (6) are appropriate for bluff bodies with flat surfaces orthogonal to the wind. For more cylindrical structures (such as the top end of a telescope), most of the force in the wind direction is produced over a smaller fraction of the cross-sectional area, reducing the effective size of the structure relative to the scale size of turbulence, and thus reducing the effective value of $\gamma$.

Given the various different expressions common in obtaining the amplitude and spectrum of force from the velocity, the clarification above is essential; this allows us to now develop the wind model appropriate for telescope structures.

\section{B. Wind Model for Telescopes}

A support structure representative of those being designed for future large optical telescopes is sketched in Fig. 1 to illustrate the structure exposed to wind turbulence above the elevation axis. The secondary mirror is supported by a tripod or quadrupod, rather than the spider-supported system common in the current generation of optical telescopes. While the support members may have significant crosssectional area, the spatial decorrelation of pressure along their length significantly reduces the net unsteady force on them.

The following assumptions greatly simplify the description of the response and are validated by the full integrated model.

i. Image jitter caused by unsteady wind forces is dominated by the loads on the upper part of the telescope (top end and structural members) rather than by forces across the primary mirror. This is a result of lower wind speeds near the primary (assuming a well-designed enclosure).

ii. For a well-designed telescope structure, the impact of these loads is almost entirely image motion (optical tip/tilt); the effect that the wind on the top end has on either optical misalignment or M1 segment displacements is small in comparison. (This was not true for some telescope structural designs where the load path from the secondary support passed through the primary mirror cell [11].)

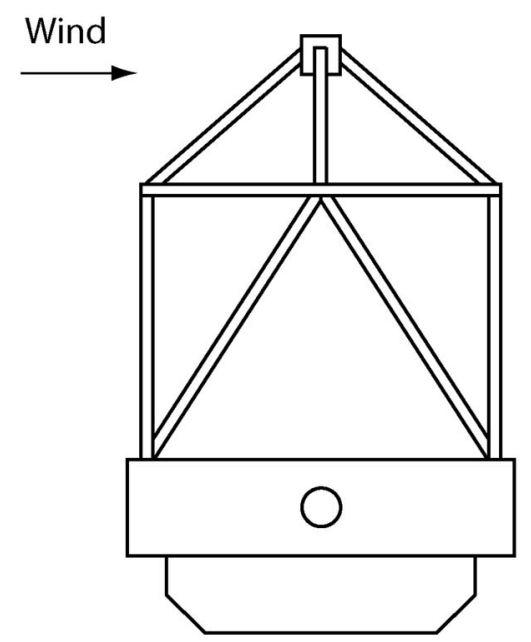

Fig. 1. Representative extremely large telescope design showing upper structural members relevant for wind loads. The top unit is supported by a quadrupod or tripod structure that connects to a ring between the elevation axis and the top unit. Only the members above the ring will be used in the simplified model. 
iii. Most of the image jitter comes from the MCS residual rather than structural deformation (either quasi-static or resonant), and, therefore, representing the disturbance as a wind torque about the drive axes, or equivalently as a single force at the top end of the telescope (rather than a distributed load) is a reasonable approximation.

iv. The azimuth axis has higher inertia and lower moment arm for wind loads and, thus, will typically have lower response. Hence, only the elevation-axis response needs to be included; the approach here can easily be extended to also predict the azimuth response.

v. The peak torque response occurs near the $-3 \mathrm{~dB}$ bandwidth of the MCS, roughly $0.5 \mathrm{~Hz}$ for extremely large telescopes. This means that the model does not need to be accurate either at very low frequencies (which are adequately controlled by the MCS) or at very high frequencies (where the inertial response of a massive structure is small).

vi. In particular, low-frequency wind loads due to variations in external wind speed and orientation are adequately controlled, and only the loads due to turbulence need to be estimated. This is also the dominant source of unsteadiness measured inside the Gemini enclosure [4].

vii. Some model of spatial decorrelation is essential for capturing the wind response correctly. For mean wind speed $\bar{u}$ and at frequency $f$, the correlation length is a fraction $1 / \gamma$ of the frozen-turbulence wavelength $\lambda=\bar{u} / f$, with $\gamma=2$ for a long slender structural member.

viii. From (v) and (vii), and for wind speeds inside the telescope enclosure of order $2 \mathrm{~m} / \mathrm{s}$ (for example), the correlation length is of order $2 \mathrm{~m}$ at the peak response frequency of $0.5 \mathrm{~Hz}$. This means that forces on different structural members that are separated by more than this dimension are uncorrelated and can be added in quadrature without significant error. The torque spectra can be estimated separately for the top end of the telescope and for each structural member above the elevation axis and added in the frequency domain.

ix. Because both the wind speed and the moment arm decrease with distance away from the top end, most of the response is due to the top-end area and uppermost structural members, and the remaining structure above the elevation axis can be ignored. Assuming that the wind speed on the uppermost members is the same as the wind speed at the top end reduces the number of parameters required to characterize wind loads without significant error.

$\mathrm{x}$. Wind speed and pressure follow von Karman power spectra. This is well validated by measurements at Gemini, in wind-tunnel testing, and in CFD; see [4] for a summary.

xi. The forces can be obtained from the projected area, an estimated drag coefficient, and the dynamic pressure.

Given the above assumptions, the following equations describe the spectrum of the unsteady wind torque about the elevation axis. Nomenclature and representative parameter values (for TMT) are given in Table 1 . For ease of scaling, the mean velocity at the telescope top end $\bar{u}=\alpha U_{\infty}$ is defined as the product of the external wind velocity $U_{\infty}$ and the reduction factor $\alpha$ that is provided by the enclosure.

Force spectra are defined for the telescope top end (subscript M2), and the structure that supports it (subscript $s$ ). The latter has $n_{s}$ identical structural members (three for a tripod support; see Fig. 1 for an illustration). The spectra, as a function of frequency $f$, are

$$
\begin{gathered}
\Psi_{M 2}(f)=F_{M 2}^{2} \Phi_{p}(f) H_{M 2}(f) \\
\Psi_{s}(f)=n_{s} F_{s}^{2} \Phi_{p}(f) H_{s}(f) .
\end{gathered}
$$

Table 1. Parameters Affecting Wind Force on Structure ${ }^{a}$

\begin{tabular}{llc}
\hline Parameter & \multicolumn{1}{c}{ Meaning } & Value \\
\hline$L_{0}$ & Outer scale [defined by Eq. (3) or (5) ] & $0.9 \mathrm{mg} / \mathrm{m}^{3}$ \\
$\rho$ & Air density & $8 \mathrm{~m} / \mathrm{s}$ \\
$U_{\infty}$ & External mean wind speed & 0.25 \\
$\alpha$ & Ratio of mean wind speed at top end to external & 0.5 \\
$\mathcal{I}$ & Turbulence intensity inside enclosure & 1.5 \\
$C_{D, M 2}$ & Drag coefficient of top end structure & 2 \\
$C_{D, s}$ & Drag coefficient of upper structural elements & $10 \mathrm{~m}^{2}$ \\
$A_{M 2}$ & Transverse cross-sectional top-end area & $13.5 \mathrm{~m}$ \\
$\ell_{x}$ & Projected length of each upper structural element & $0.6 \mathrm{~m}$ \\
$\ell_{y}$ & Width of each upper structural element & $25 \mathrm{~m}$ \\
$h_{M 2}$ & Mean height of top end above elevation axis & $17.5 \mathrm{~m}$ \\
$h_{s}$ & Mean height of upper structure above elevation axis & 3 \\
$n_{s}$ & Number of identical elements in upper structure & 2 \\
$\gamma_{s}$ & Number of uncorrelated turbulence cells per wavelength, structure & 1 \\
$\gamma_{M 2}$ & Same as above for M2 & \\
\hline
\end{tabular}

${ }^{a}$ Representative values (taken from TMT) are provided to indicate the point in parameter space at which the model herein is verified. The external wind speed, of course, varies, and the internal wind speed varies with orientation, even for a fixed enclosure design. Note that with a tripod structure, the projected area facing the wind is a factor of $\sqrt{2}$ smaller than the actual area, independent of orientation (assuming that the three support members add in quadrature, and neglecting the on-axis area of each member). 
$F_{M 2}$ and $F_{s}$ are the net unsteady forces if the pressure was correlated across the top end or a structural member, respectively, of the form

$$
F=C_{D} A \frac{1}{2} \rho u_{\mathrm{eff}}^{2},
$$

using the appropriate cross-sectional area and drag coefficient and with $u_{\text {eff }}$ from Eq. $(2)$. $\Phi_{p}(f)$ is the pressure spectrum from Eq. (5) with $p_{\text {rms }}$ set to 1 (since this is already accounted for in the force). The spectrum depends only on the local mean wind speed and the outer scale of turbulence. $H(f)$ is the effect of the spatial decorrelation over the projected area of the structural member in both length and width, from Eq. (6).

The total torque spectrum about the elevation axis is obtained by adding the force spectra multiplied by the relevant moment arm squared

$$
\Psi_{T}(f)=h_{s}^{2} \Psi_{s}(f)+h_{M 2}^{2} \Psi_{M 2}(f) .
$$

It is clear how to extend this to include all of the structural members above the elevation axis in Fig. 1; they are neglected here for simplicity in parameterization.

\section{Response Model}

Image motion is related to wind torque through the torque rejection transfer function $R(s)$, which depends on the telescope structure and the control law. Hence

$$
\theta_{\mathrm{rms}}^{2}=\int_{f=0}^{\infty} \Psi_{T}(f)|R(f)|^{2} \mathrm{~d} f,
$$

where $\Psi_{T}(f)$ is defined in Eq. (11), $\theta_{\text {rms }}$ is the rms image motion, and $R(f)$ is the transfer function $R(s)$ evaluated at $s=j \omega=j 2 \pi f$.

The closed-loop torque response due to the finite mount control bandwidth can be computed from the open-loop torque response $G(s)$ and the controller transfer function $K(s)$ as $R_{\mathrm{MC}}(s)=G /(1+G K)$. The achievable control bandwidth on a given structure depends on the details of the structural dynamics, including damping. However, the low-frequency torque response is adequately represented if the telescope is modeled only with the inertial response $G(s)=$ $1 /\left(J s^{2}\right)$, where $J$ is the moment of inertia about the elevation axis.

Rather than using the exact controller transfer function designed for a particular structure, the response can be parameterized in terms of the control bandwidth $f_{c}$ by assuming a proportional-integralderivative (PID) control law with well-damped zeros. The relevant bandwidth is the $-3 \mathrm{~dB}$ point on the sensitivity $S=1 /(1+G K)$. If

$$
K(s)=k_{i} / s+k_{p}+k_{d} s=J \beta \omega_{k}\left(s^{2}+\sqrt{2} \omega_{k} s+\omega_{k}^{2}\right) / s
$$

for PID gains $k_{i}, k_{p}$, and $k_{d}$, then choosing $\omega_{k}=$ $\left(2 \pi f_{c}\right) / 2$ and $\beta=3$ gives the desired bandwidth and a $60^{\circ}$ phase margin.

The quasi-static effect of the telescope structural compliance can also be added to the mount response computed above. Compliance can be obtained from the structural and optical model by applying a unit static force on the top end with the control loops closed (or with a locked-rotor model) and computing the resulting image motion. For consistency with the units of $R$, this can be scaled by the moment arm to give the image motion per unit torque, $R_{S C}$. Since it is the same wind force that produces both the elevation-axis rotation and the structural bending, the combined image motion adds linearly, not in quadrature.

The effect of an optical guide loop can be included by high-pass filtering of the response spectrum. Assuming integral control and a $60^{\circ}$ phase margin due to time delay, then the sensitivity transfer function for an optical guide loop with bandwidth $f_{g}$ can be modeled as

$$
F(s)=\frac{s}{s+\omega_{g} e^{-s \tau / \omega_{g}}},
$$

where $\omega_{g}=2 \pi f_{g}$ and $\tau=1 / 12$ (delay normalized by bandwidth). Then $R(s)$ in Eq. (12) is

$$
R=F\left(R_{S C}+\frac{G}{1+G K}\right) .
$$

At a relatively low bandwidth (e.g., for the parameters in Table 2), the guide loop has minimal effect on the response due to the finite mount control bandwidth, but it significantly reduces the contribution due to structural compliance. Thus, even at a low bandwidth, the presence of the guide loop means that it is a reasonable assumption to ignore the compliance. Thus, while the simulations in Section 4 use the parameterization in Eq. (15), choosing $R_{S C}=0$ and $F=1$ would yield almost the same prediction for image jitter.

Representative parameter values (taken from TMT) are given in Table $\underline{2}$.

\section{Verification}

\section{A. Thirty Meter Telescope Example}

Wind response predictions for the TMT [16] are used as an example to validate the model presented in the previous section. The TMT uses a Calotte enclosure, which is quite different from other enclosure designs. This leads to smaller wind loads on the telescope (reflected in the nominal choice of $\alpha$ in Table 1), but it does not affect the process for evaluating wind response.

To evaluate wind response of the TMT, two different integrated models have been used, thereby providing independent cross checks of the response to wind loads. We here compare the simpler dedicated 
Table 2. Parameters Affecting Response to Wind Loads ${ }^{a}$

\begin{tabular}{llc}
\hline Parameter & \multicolumn{1}{c}{ Meaning } & Value \\
\hline$J$ & Rotational inertia & $1.4 \times 10^{8} \mathrm{~kg} \mathrm{~m}{ }^{2}$ \\
$R_{S C}$ & Static compliance (image motion per unit torque) & $3.4 \times 10^{-11} \mathrm{rad} / \mathrm{Nm}$ \\
$f_{c}$ & Elevation-axis mount control bandwidth & $0.6 \mathrm{~Hz}$ \\
$f_{g}$ & Guide loop bandwidth & $0.15 \mathrm{~Hz}$ \\
\hline
\end{tabular}

${ }^{a}$ Values (taken from TMT) are provided to indicate the point in parameter space at which the model herein is verified.

model described above with integrated models encompassing all major dynamic effects of the telescope system. The model summarized below originates from a generic set of simulation tools first applied for the proposed Euro50 extremely large telescope $[17,18]$ and then later expanded and applied to the European Extremely Large Telescope (E-ELT) [8]. A few specific aspects have been explored in more detail in a separate integrated model described in $[9,10]$; these include the effect of the guide loop, and a more detailed mount control design to maintain bandwidth with lower structural damping.

\section{B. Integrated Model}

A block diagram of the integrated model is shown in Fig. 2; the second integrated model is similar but neglects details of the segment dynamics and control. The structure submodel originates from an ANSYS finite element model of the telescope that has been exported to the integrated model environment (MATLAB). A model reduction has been performed by modal truncation retaining 5000 modes, corresponding to eigenfrequencies below $111 \mathrm{~Hz}$, and the structure converted to state-space form (for input $w$ and output $y, \dot{x}=\mathbf{A} x+\mathbf{B} w, y=\mathbf{C} x$, where the matrices $\mathbf{A}, \mathbf{B}$, and $\mathbf{C}$ characterize the structure). Typical values for the modal damping ratio $\zeta$ lie in the range 0.005-0.02 (e.g., Keck damping is in this range [19]). The choice of damping ratio does not have a significant effect directly on the wind response, but indirectly affects the response because it influences the maximum mount control servo bandwidth that can be used. The first integrated model uses $\zeta=0.02$, which allows relatively simple servo designs to be used, while the second model uses a more conservative value of $\zeta=0.005$ along with a more detailed servo design to maintain comparable bandwidth [20].

The main servos of the MCS have been modele $\bar{a}$ s conventional cascade controllers, including proportional-integral (PI) velocity and position loop controllers with roll-off above $1 \mathrm{~Hz}$ for elevation and $2 \mathrm{~Hz}$ for azimuth, and with a bandwidth $(-3 \mathrm{~dB}$ sensitivity) of $0.43 \mathrm{~Hz}$ for elevation and $0.3 \mathrm{~Hz}$ for azimuth. The controllers are similar to those described in Subsection 2.C. A bandwidth of $0.6 \mathrm{~Hz}$ in both elevation and azimuth is obtained with the more detailed servo design.

The optical system, also shown in Fig. 2, has been modeled using sensitivity matrices defining focal plane spot displacements as a linear function of the displacements of all mirror elements, including each of the 492 segments of the primary mirror.
The resulting pointing error of the telescope is determined as the average of the spot centroid displacements in two mutually perpendicular directions.

The upper part of Fig. 2 relates to the primary mirror and to segment control; these dynamics are included here to validate that they do not affect image jitter (they are, of course, relevant for predicting other aspects of the response). Each of the 492 segments has been modeled as a rigid body with translation in 5 degrees of freedom in a coordinate system with origin at the nominal vertex of the primary, with the $z$ axis aligned with the tube axis and pointing toward the sky. Segment rotation about the $z$ axis is neglected. Static compliance of the mirror segment supports has been included, together with models of each of the $3 \times 492=1476$ actuators; these are modeled as $10 \mathrm{~Hz}$ bandwidth servomechanisms with cascade-coupled velocity and position PI controllers. The segment control system generates commands to the actuators with feedback from edge sensors. The 2772 edge sensor signals are related to the segment displacements through an interaction matrix that can be determined from geometry. The segment control system uses a modal controller based on a singular value decomposition. Each of the 1473 observable modes is controlled by a separate integral controller with a $1 \mathrm{~Hz}$ bandwidth, with the exception of the global radius-of-curvature mode of the mirror, which is left uncontrolled in the present model.

A thorough analysis of wind loads on all of the upper members of the telescope has been conducted [10], including the effects of reduced wind speed further from the aperture, as well as reduced moment arm. The resulting wind spectrum is almost identical to the simpler model developed herein, as shown in Fig. $\underline{3}$, and only the simpler version is used

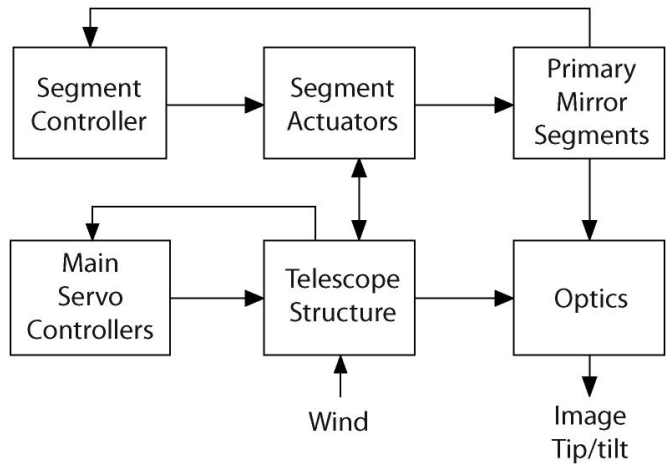

Fig. 2. Block diagram of the full integrated model used for validation. 
in validating that the simple prediction of the wind response developed herein matches that of the full integrated models.

\section{Comparisons}

Figure 4 validates the approximate torque response prediction by comparing it with the prediction made with the full structural model and the more detailed servo design. Also shown is the effect of increased mount control bandwidth and the structural static compliance for comparison. With the nominal control bandwidth, the static contribution is larger below roughly $0.05 \mathrm{~Hz}$. Thus, if there is an optical guide loop with at least this bandwidth, the torque response will be dominated by the mount control. At high frequencies, the structural dynamics can be significant, but there is negligible wind energy, as seen by Fig. 3 .

Figures 5 and 6 compare the image jitter spectra computed from the integrated models to the prediction using the simple approach presented herein, using all of the approximations, except that the same mount control and guide feedback laws are used as in the integrated model they are compared with. These demonstrate that the additional effects included in the full integrated model are not essential to predict image jitter: the small differences in the spectra in Fig. 7 between the integrated model and the approximate model are not due to missing physics, but simply due to the approximation of the control laws. Note that the image jitter response in these figures differs slightly from the "nominal" performance in the next section because the parameter values in Table $\underline{2}$ are rounded off for simplicity, while the exact values are used to compare with the full integrated model.

It is also worth noting that the approximations herein break down at higher frequencies where the response is dominated by the structural dynamics, rather than by the mount control residual. However,

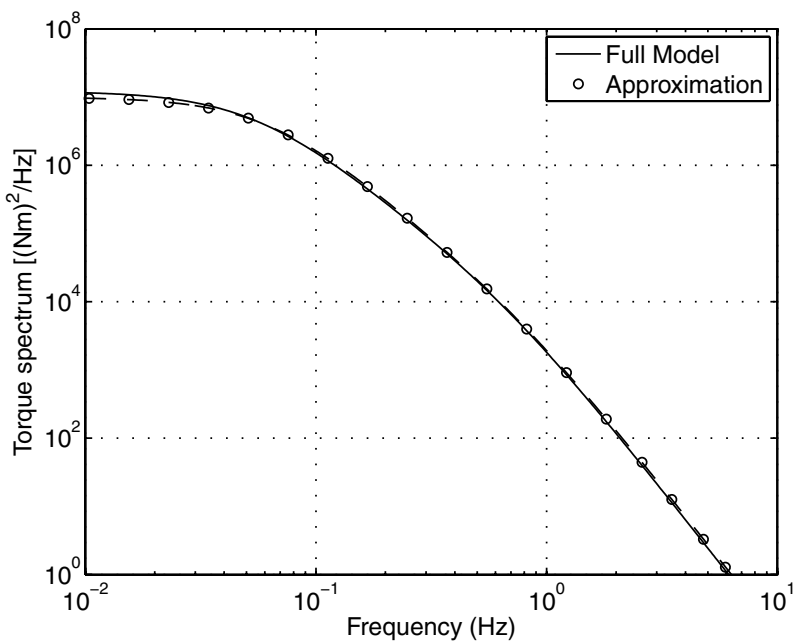

Fig. 3. Comparison between full wind spectrum (computed for TMT, with forces on all structural members above the elevation axis) and approximate wind spectrum [Eq. (11)], with parameters as in Table $\underline{1}$.

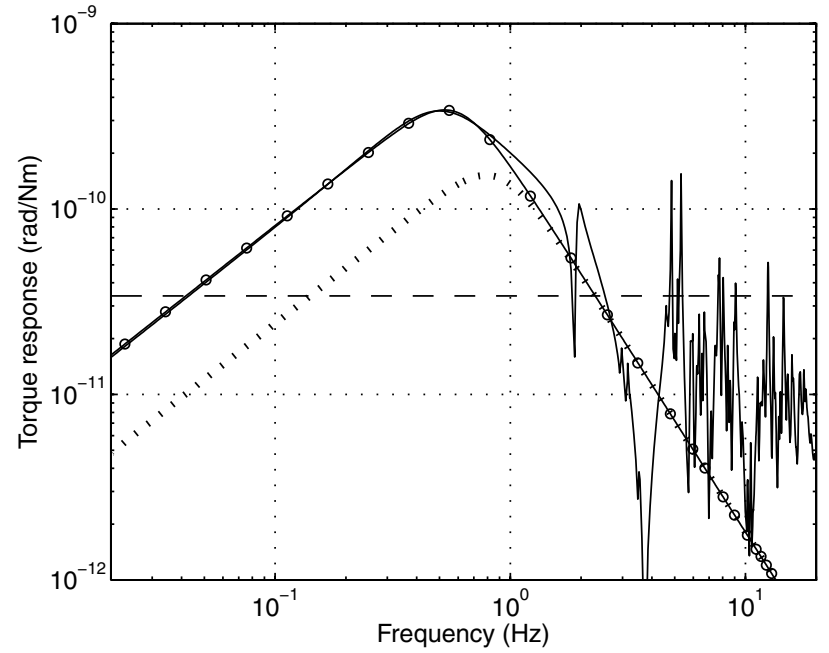

Fig. 4. Comparison between full mount control torque response and simple approximation with the same bandwidth (circles). Parameters are in Table 2; structural damping in the full model here is $0.5 \%$. The horizontal dashed line is the static structural compliance for comparison. The dotted curve corresponds to a $50 \%$ increase in control bandwidth for the approximation.

since there is relatively little wind energy at these frequencies, the overall image jitter is accurately predicted. If the higher frequency content needs to be understood (e.g., for adaptive optics simulations), then the response including at least a few relevant structural modes would be required.

\section{Spatial Decorrelation}

The importance of using the correct spatial decorrelation model is illustrated in Fig. 8 for the telescope top end and structural members separately. Each plot uses either the (correct) spectrum from Eqs. (5) and (6) or the pressure spectrum with a $-5 / 3$ exponent and the aerodynamic attenuation factor in (7).

For the low-aspect-ratio telescope top end, the main difference results from using $\gamma_{M 2}=1$ in

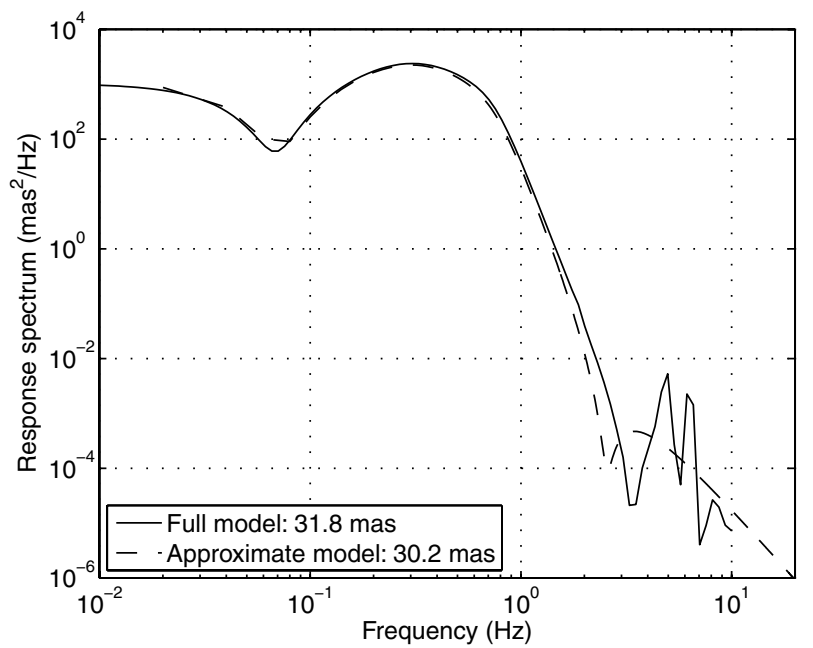

Fig. 5. Comparison of response spectrum for integrated model of TMT (solid curve) and using the simple model (dashed curve) but with the same mount control law as the integrated model. The image jitter differs by roughly $5 \%$. 


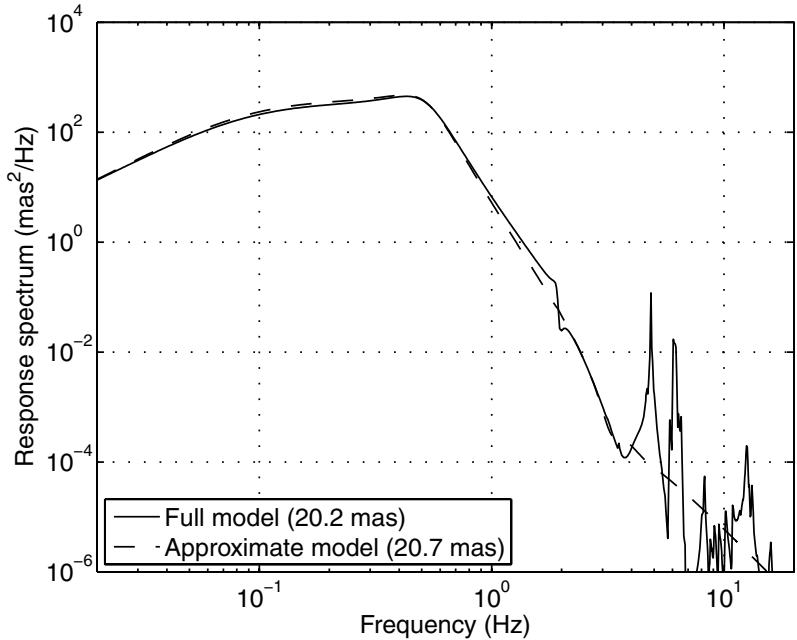

Fig. 6. Same as Fig. 5 , but with the second integrated model that also includes the guide control. (The decrease in response relative to Fig. 5 is due to the higher control bandwidth, not the presence of the guide law.)

Eq. (6), while using the fixed value of 2 in Eq. (7) in order to compare to the result that would be obtained if the source of the aerodynamic attenuation is not understood. The correct value for this nonrectangular structure is not known, but is certainly less than 2 since most of the force is generated over a fraction of the projected area. Using the $-5 / 3$ exponent shifts energy from low to higher frequencies, while the greater spatial decorrelation decreases energy at high frequencies; the net effect is a reduction in energy everywhere and a $25 \%$ reduction in the predicted response due to loads on the top end.

For the high-aspect-ratio structural members supporting the top end, the correct spatial decorrelation reduces the high-frequency force relative to what would be predicted from Eq. (7), which parameterizes the decorrelation using only the area. Combined with the $-5 / 3$ assumption that shifts energy to higher frequencies, the typical approach yields more than a $40 \%$ increase in the predicted response due to the

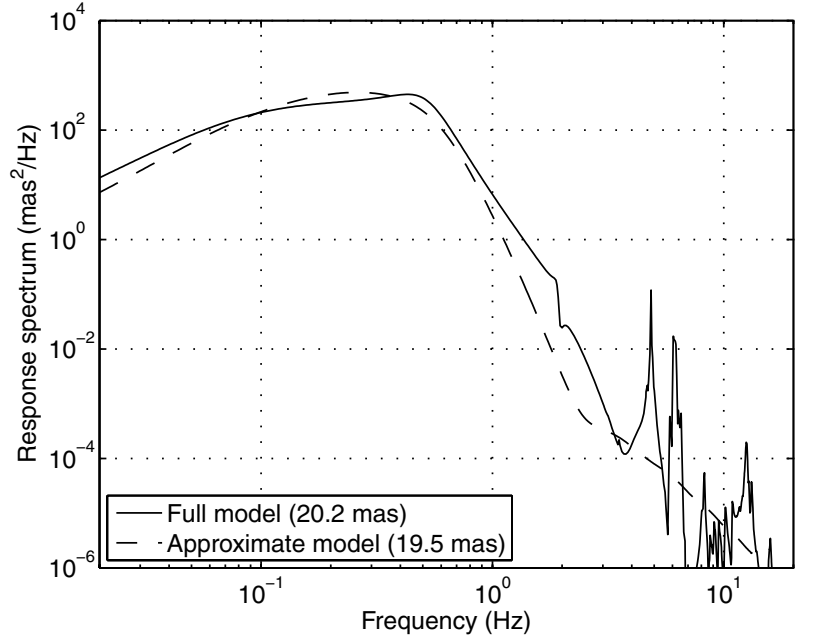

Fig. 7. Same as Fig. 6 , but using the approximate mount control law in computing the response for the simple model. The image jitter differs by roughly $3.5 \%$.

structural members. This changes the situation from the response being dominated by loads on the top end, to the response being dominated by loads on the structural members, which clearly would influence design choices.

In addition to different predictions due to different assumptions about spectra, the second term in Eq. (2), which is typically ignored, leads to a $6 \%$ increase in the response at a turbulence intensity $\mathcal{I}=0.5$.

\section{Parametric Dependence}

It is not necessary to compute the parametric dependency with respect to all of the parameters in Tables 1 and 2 , since the effects of some of them are quite obvious (e.g., air density, rotational inertia, or moment arm). The scaling of the response to turbulence intensity follows from Eq. (2) and is almost linear.

Figures 9 and 10 plot the image jitter predicted by this model as a function of selected wind, structure, and control parameters:
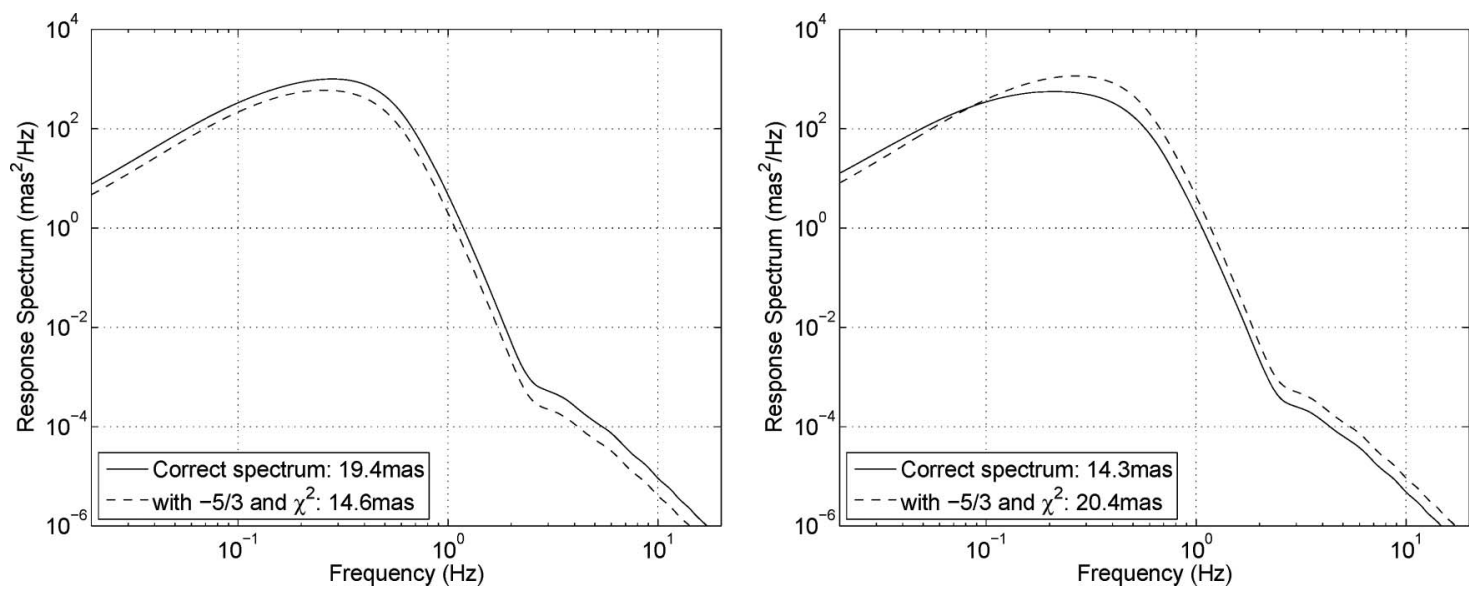

Fig. 8. Effect of spatial decorrelation assumptions, either the correct ( $-7 / 3$ slope) pressure spectrum and decorrelation $H(f)$ separately in two axes, or $-5 / 3$ exponent on pressure spectrum and standard aerodynamic attenuation $\chi^{2}(f)$. Left, effect on response due to loads on top-end structure. Right, effect on response due to loads on upper structural members. 
- External wind speed $U_{\infty}$. While it is clear that the response should increase at least quadratically (since the wind force does), the actual increase is closer to $U_{\infty}^{3}$ because higher wind speed implies higher frequency content and, thus, increases the fraction of wind energy at the frequencies at which the system responds the most.

- Enclosure reduction factor (ratio of internal mean wind speed to external, keeping the turbulence intensity the same). While the effect of this should be the same as changing the external velocity, this is more directly connected to the design of the enclosure, while the external wind speed is essentially fixed by the choice of site, and it is therefore useful to visualize the dependence on this parameter.

- Outer scale of turbulence. The nominal outer scale of turbulence is set by the aperture dimension (the source of the turbulence); halving the outer scale with all other parameters constant results in a roughly $50 \%$ increase in response due to the increased content at higher frequencies.

- Spatial decorrelation length factor $\gamma$. While it is critical that this is nonzero (otherwise the assumption that most of the structural support members in Fig. 1 can be ignored is not valid), the actual response $\bar{d}$ oes not depend strongly on either the decorr-
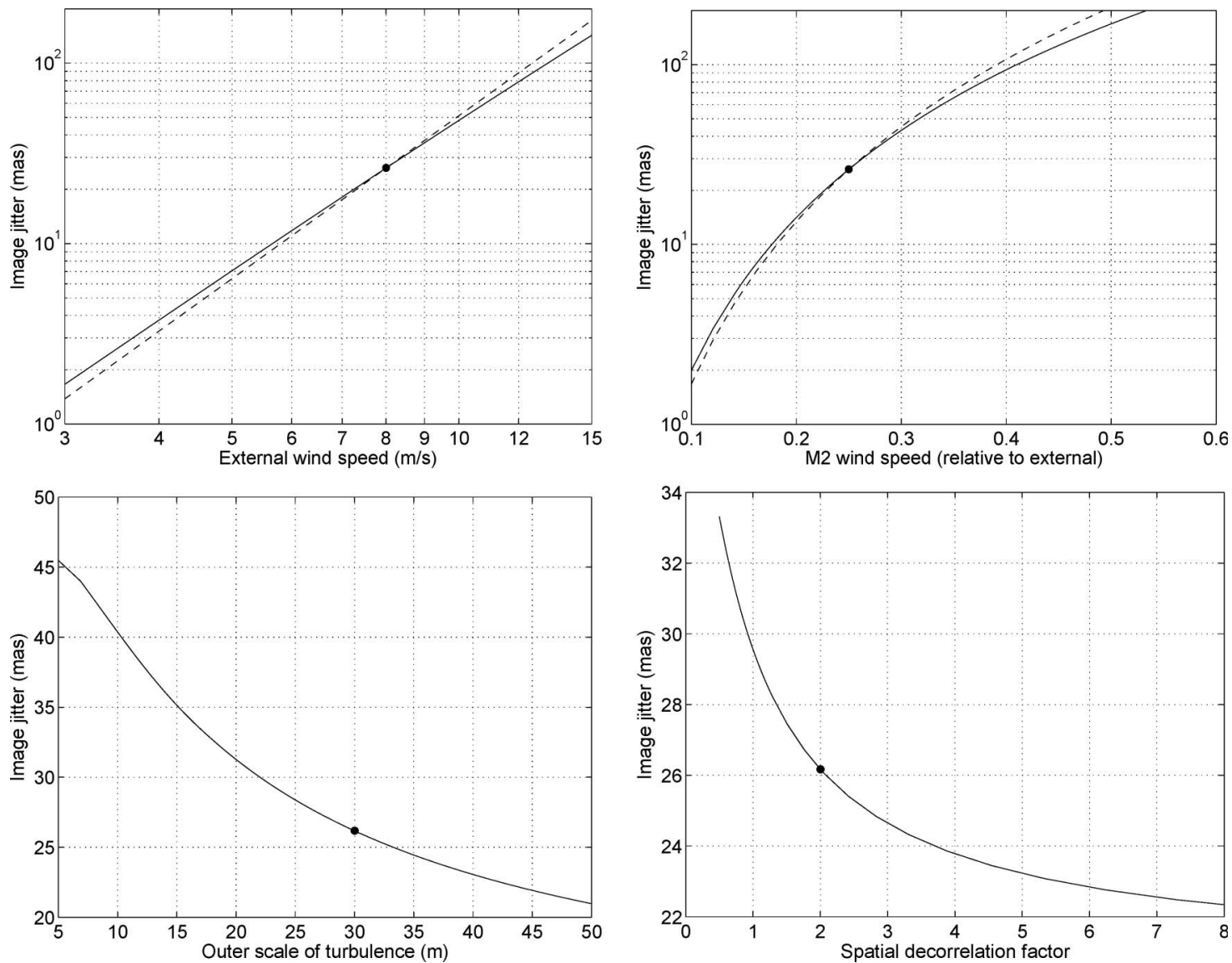

Fig. 9. Image jitter sensitivity to wind parameters, keeping all other parameters constant at the nominal values given in Tables 1 and 2 (nominal performance indicated with circle). Top left, varying external wind speed; top right, varying internal wind speed. (The dashed curve in both plots is proportional to wind speed cubed.) Bottom left, varying outer scale of turbulence; bottom right, varying spatial decorrelation length scale over the support members. 

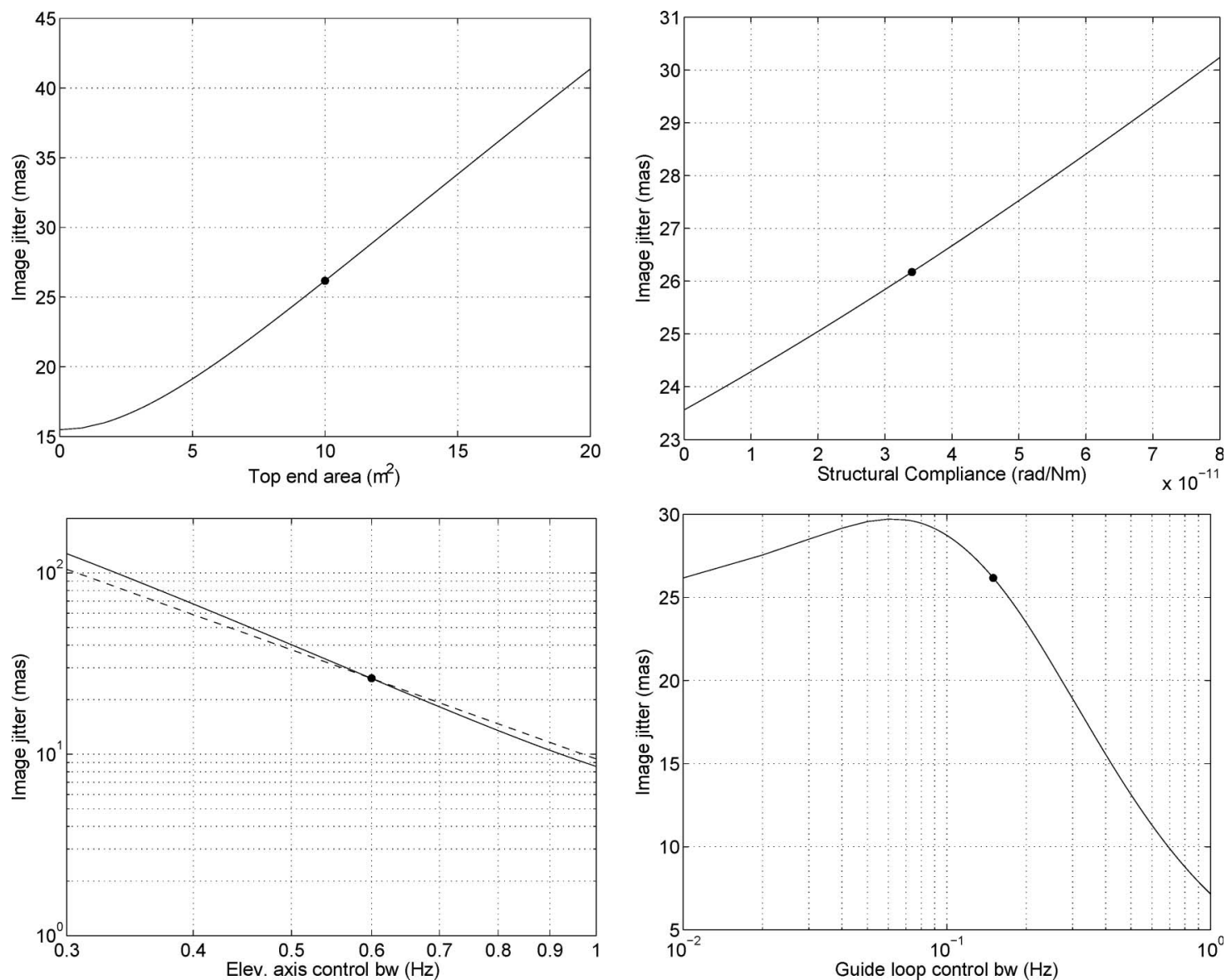

Fig. 10. Image jitter sensitivity to structure and control parameters, keeping all other parameters constant at the nominal values given in Tables $\underline{1}$ and $\underline{2}$ (nominal performance indicated with circle). Top left, varying top-end area; top right, varying static compliance. Bottom left, varying elevation axis control bandwidth (with $f_{c}^{-2}$ plotted); bottom right, varying guide loop control bandwidth.

- Guide loop bandwidth. The assumed form for the guide loop sensitivity includes some amplification at frequencies just above the bandwidth. Thus,

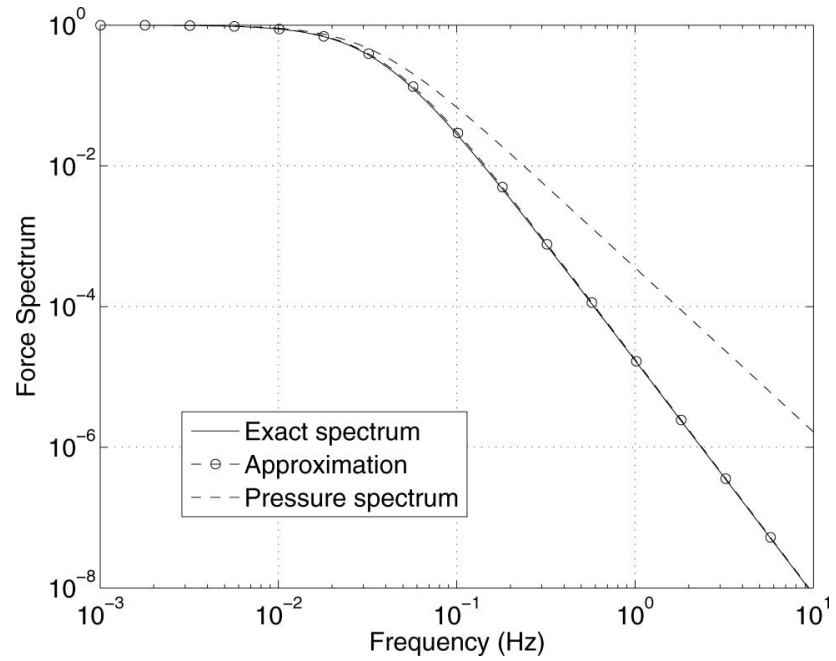

Fig. 11. Comparison of exact integral and fit for the normalized force spectrum on a structure of length $\ell_{x}$ perpendicular to the wind and comparison with normalized pressure spectrum. The outer scale used in this figure was $L_{0}=30 \mathrm{~m}$, structure length $\ell_{x}=10 \mathrm{~m}$, and wind speed $\bar{u}=1 \mathrm{~m} / \mathrm{s}$. depending on the wind energy at frequencies rejected by the guide loop compared with the energy at frequencies amplified by the guide loop, the closed-loop response can increase for some choices of bandwidth. The nominal bandwidth of $0.15 \mathrm{~Hz}$ is sufficient to reject most of the quasi-static structural response, at the expense of an almost comparable increase in the response due to the mount control. Increasing the guide loop bandwidth beyond the mount control bandwidth results in a significant reduction in image motion. Note that if the guiding is implemented with the mount control drives, then the nominal choice of roughly one-fourth the MCS bandwidth is the maximum feasible; higher bandwidth would require tip/ tilt correction elsewhere in the telescope.

\section{Conclusions}

Image jitter due to unsteady wind loads inside the enclosure of a ground-based optical telescope can be accurately predicted using a relatively simple model. An accurate model of the spatial decorrelation of the pressure (as opposed to the usual aerodynamic attenuation factor) is essential to understanding wind forces on long slender structural members supporting the top end of the telescope. The dominant contribution to the response is from the finite band- 
width of the MCS, and the elevation axis in particular, and not from either quasi-static or dynamic structural deformation. The mount response is small at both low frequencies where the loop gain is high and at high frequencies where the inertial effect is high and has a peak response near the mount control bandwidth.

The image jitter response scales nearly with the wind speed cubed, because in addition to the pressure (and, hence, force) scaling with the square of the speed, higher speeds mean shorter convective time constants and, thus, higher frequency excitation, resulting in more wind energy at the frequencies where the system responds. The image jitter scales roughly with the -2 power of the mount control bandwidth; higher bandwidth means both that the peak response frequency is higher (where there is less wind energy) and also that the response at the peak frequency is lower.

The image jitter scaling with parameters such as density, cross-sectional area, moment arm, or inertia is self-evident. The response does depend on having a model of the spatial decorrelation of pressure, as this greatly decreases the unsteady forces on long slender structural members at frequencies at which the MCS responds. However, because any realistic model of the spatial decorrelation results in the concentrated cross-sectional area at the top end of the telescope being the dominant contributor to image jitter, the response is not strongly sensitive to the assumed spatial correlation length.

The insights gained both from understanding the relevant physics that determine the telescope image jitter and from understanding which parameters the response is most sensitive to are essential in designing all elements of the observatory that contribute to the wind response: the enclosure, the structure, and the control systems.

\section{Appendix A: Frozen Turbulence Decorrelation Calculation}

We want to know the net force on the structure as a function of frequency, and not simply the pressure. The frequency-dependent spatial decorrelation to predict the force spectrum can be obtained assuming von Karman pressure statistics and frozen turbulence. This derivation previously appeared in [10], and is similar to that in [4] but more directly applicable to the assumptions made herein regarding the geometry and wind speed characteristics.

Most structural members of the upper telescope are long and thin, so decorrelation is primarily relevant over one dimension of length $\ell_{x}$. For simplicity, consider wind speed $\bar{u}$ orthogonal to the structure, and arbitrarily label this the $x$ axis.

At a given wavenumber $k_{y}$, the force is the integral of the sinusoidally varying pressure along the structure:

$$
F=\int_{-\ell_{x} / 2}^{\ell_{x} / 2} p(y) \mathrm{d} y
$$

hence

$$
F\left(k_{y}\right)=\ell_{x}\left|p\left(k_{y}\right)\right| \frac{\sin k_{y} \ell_{x}}{k_{y} \ell_{x}} .
$$

The amplitude of the pressure is obtained from the 2$D$ von Karman pressure spectrum

$$
\Phi_{p}^{2 \mathrm{D}}(k)=\left(\frac{1}{1+\left(k / k_{0}\right)^{2}}\right)^{10 / 6}
$$

with wavenumber $k=\left(k_{x}^{2}+k_{y}^{2}\right)^{1 / 2}$ and $k_{0}=2 \pi / L_{0}$ for outer scale $L_{0}$. (The exponent in the 2-D spectrum has to differ from the 1-D spectrum in Eq. (5) so that integrating over one spatial wavenumber dimension will obtain the correct 1-D exponent.) Frozen turbulence relates the temporal frequency to the wavenumber in the wind direction, $k_{x}=2 \pi f / \bar{u}$. The total contribution at frequency $f$ is obtained by integrating over all possible wavenumbers $k_{y}$ in the orthogonal direction. Thus

$$
\Phi_{F}(f)=\ell_{x}^{2} \int_{0}^{\infty} \Phi_{p}^{2 \mathrm{D}}(k)\left[\frac{\sin k_{y} \ell_{x}}{k_{y} \ell_{x}}\right]^{2} \mathrm{~d} k_{y} .
$$

The integration can be performed numerically. The overall temporal force spectrum that results from (A1) can reasonably be approximated as the product of the 1-D von Karman pressure spectrum with corner frequency set by the turbulence outer scale and an additional attenuation factor with corner frequency that is a function of the spatial length scale of the structure. Both factors involve the same convective velocity to scale between spatial and temporal frequencies. Therefore, if one is considering structures that are small compared to the outer scale of turbulence, one can ignore the structural spectral factor.

The structural attenuation factor (on the power spectrum, not the amplitude spectrum) that is used in the fit shown in Fig. 11 is

$$
H(f)=\frac{1}{\sqrt{1+\left(f / f_{x}\right)^{2}}}
$$

with

$$
f_{x}=\frac{\bar{u}}{2 \ell_{x}} .
$$

The high-frequency attenuation relative to the pressure spectrum is a factor

$$
n=\frac{2 \ell_{x} f}{\bar{u}}=\frac{\ell_{x}}{\lambda / 2}
$$

(a factor of $\sqrt{n}$ in amplitude). This is equivalent to the force resulting from $n$ independent turbulent structures that add in quadrature, where the correlation length that defines $n$ is half the wavelength $\lambda=\bar{u} / f$. 
We are grateful to Zhiwen Wu for coding part of the wind load spectrum software of the integrated model and to Peter Linde for exporting the structural model from ANSYS to the integrated model. Konstantinos Vogiatzis at the Thirty Meter Telescope (TMT) contributed significantly to our understanding of wind loads.

This work was supported by the TMT Project. The TMT Project gratefully acknowledges the support of TMT partner institutions. They are the Association of Canadian Universities for Research in Astronomy (ACURA), the California Institute of Technology, and the University of California. This work was supported as well by the Gordon and Betty Moore Foundation, the Canada Foundation for Innovation, the Ontario Ministry of Research and Innovation, the National Research Council of Canada/Conseil National Recherches Canada (NRC/CNRC), the Natural Sciences and Engineering Research Council of Canada, the British Columbia Knowledge Development Fund, the Association of Universities for Research in Astronomy (AURA), and the United States National Science Foundation (NSF).

\section{References}

1. F. Forbes and G. Gabor, "Wind loading of large astronomical telescopes," Proc. SPIE 332, 198-205 (1982).

2. M. Ravensbergen, "Main axes servo systems of the VLT," Proc. SPIE 2199, 997-1005 (1994).

3. T. E. Andersen, A. Ardeberg, H. Riewaldt, M. Lastiwka, N. Quinlan, K. McNamara, X. Wang, A. Enmark, M. OwnerPetersen, A. Shearer, C. Fan, and D. Moraru, "Status of the Euro50 project," Proc. SPIE 5489, 407-416 (2004).

4. D. G. MacMynowski, K. Vogiatzis, G. Z. Angeli, J. Fitzsimmons, and J. Nelson, "Wind loads on ground-based telescopes," Appl. Opt. 45, 7912-7923 (2006).

5. M. Quattri, R. Haase, M. B. Cabrera, E. F. Monasterio, R. Codina, M. G. Hernandez, and M. Reyes, "Wind turbulence structure inside telescope enclosures," Proc. SPIE 7017, 701700 (2008).
6. K. Vogiatzis, "Advances in aerothermal modeling for TMT," Proc. SPIE 70170, 70170Q (2008).

7. M. Mamou, A. Tahi, A. Benmeddour, K. R. Cooper, I. Abdallah, M. Khalid, and J. Fitzsimmons, "Computational fluid dynamic simulations and wind tunnel measurements of unsteady wind loads on a scaled model of a very large optical telescope: a comparative study," J. Wind Eng. Ind. Aerodyn. 96, 257-288 (2008).

8. T. E. Andersen, A. Enmark, P. Linde, M. Owner-Petersen, A. Sjöström, F. Koch, M. Müller, L. Noethe, and B. Sedghi, "An integrated model of the European ELT," Proc. SPIE 7017, 70170S (2008).

9. D. G. MacMynowski, C. Blaurock, G. Z. Angeli, and K. Vogiatzis, "Modeling wind-buffeting of the Thirty Meter Telescope," Proc. SPIE 6271, 62710M (2006).

10. D. G. MacMynowski, C. Blaurock, and G. Z. Angeli, "Dynamic analysis of TMT," Proc. SPIE 7017, 70170W (2008).

11. S. Padin and W. Davison, "Model of image degradation due to wind buffeting on an extremely large telescope," Appl. Opt. 43, 592-600 (2004).

12. T. von Kármán, "Progress in the statistical theory of turbulence," Proc. Natl. Acad. Sci. 34, 530-539 (1948).

13. E. Simiu and R. H. Scanlan, Wind Effects on Structures: An Introduction to Wind Engineering (Wiley, 1986).

14. G. K. Batchelor, The Theory of Homogeneous Turbulence (Cambridge U. Press, 1953).

15. P. Y. Bely, The Design and Construction of Large Optical Telescopes (Springer-Verlag, 2003).

16. J. Nelson and G. H. Sanders, "The status of the Thirty Meter Telescope project," Proc. SPIE 7012, 70121A (2008).

17. T. Andersen, M. Owner-Petersen, and H. Riewaldt, "Integrated simulation model of the Euro50," Proc. SPIE 4757, 84-92 (2002).

18. T. E. Andersen, M. T. Browne, A. Enmark, D. Moraru, M. Owner-Petersen, and $\mathrm{H}$. Riewaldt, "Integrated modeling of the Euro50," Proc. SPIE 5382, 366-378 (2004).

19. M. Sirota, P. M. Thompson, and H. R. Jex, "Azimuth and elevation servo performance of the W. M. Keck Telescope," Proc. SPIE 2199, 126-141 (1994).

20. P. M. Thompson, D. G. MacMynowski, and M. J. Sirota, "Analysis of the TMT mount control system," Proc. SPIE 7012, 7012P (2008). 